\title{
Richard Tilly Nachruf auf Toni Pierenkemper (17. Oktober 1944 bis 19. Juli 2019)
}

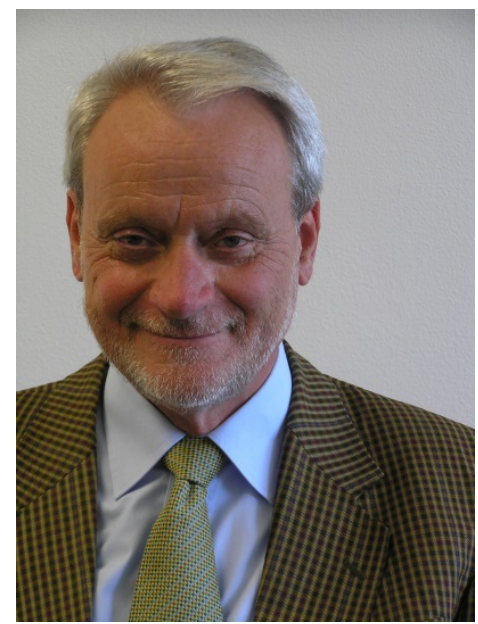

Prof. Dr. Toni Pierenkemper

Mit dem überraschenden Tod von Toni Pierenkemper am 19. Juli 2019 ist eine der wichtigen Stimmen der deutschen Wirtschafts- und Sozialgeschichte verstummt. Viele Kollegen verloren dabei einen guten Freund und geschätzten Gesprächspartner. Hier gilt es, seine Lebenswirkung als Forscher und akademischer Lehrer zu würdigen.

Geboren 1944 in Wiedenbrück, Westfalen, aufgewachsen in Münster, aber als Achtjähriger bereits mutterlos, hatte Toni Pierenkemper keinen leichten Start ins Leben. Vielleicht deshalb kam er über eine ungewöhnliche Route zur Universität: Sie führte ihn auf dem zweiten Bildungsweg über eine Lehre als Maschinenschlosser, die Berufsaufbauschule und das Overberg-Kolleg zum Abitur. Von 1967 bis 1972 studierte er dann Volkswirtschaftslehre und Soziologie an der Universität Münster. In den Jahren 1969 bis 1970 schrieb er sich mit derselben Fächerkombination an der London School of Economics in Großbritannien ein. Dort hatte er Kontakt mit Charlotte Erickson, Verfasserin einer vergleichenden Studie über britische Unternehmer. An diesem Thema zeigte er schon damals Interesse. 
1972 schloss er das wirtschaftswissenschaftliche Studium als DiplomVolkswirt ab, 1975 folgte der M.A. in Soziologie. Von 1970 bis 1984 blieb Toni Pierenkemper als wissenschaftlicher Mitarbeiter beziehungsweise Assistent an meinem Lehrstuhl. 1977 promovierte er mit einer Studie über westfälische Schwerindustrielle - eine Arbeit, die Maßstäbe setzte und in jenen Jahren als Vorbild für Unternehmerforschung diente. Nach der Promotion widmete er sich der Arbeitsmarktforschung, insbesondere den deutschen Angestellten im Kaiserreich. Seine Habilitationsschrift wurde 1984 von der Wirtschaftswissenschaftlichen Fakultät in Münster angenommen. In der Münsteraner Fakultät wirkte er anschließend - nun berufen zum C-2-Professor für Wirtschafts- und Sozialgeschichte - in Lehre und Forschung mit.

1989 erhielt er einen Ruf auf den Lehrstuhl für Wirtschafts- und Sozialgeschichte an der Universität des Saarlandes in Saarbrücken. Nach einem Jahr dort folgte er einem Ruf auf den Lehrstuhl für Wirtschafts- und Sozialgeschichte an der Universität Frankfurt, wo er bis 1997 blieb - unterbrochen in den Jahren 1993 bis 1994 durch die Wahrnehmung einer Visiting Professorship an der Georgetown University in Washington D.C. Schließlich nahm er 1997 einen Ruf auf den Lehrstuhl für Wirtschafts- und Sozialgeschichte an der Universität Köln an, wo er auch Direktor des Seminars für Wirtschafts- und Sozialgeschichte wurde. Hier blieb er bis zu seiner Emeritierung im Frühjahr 2010.

Das wissenschaftliche Werk Pierenkempers ist außerordentlich breit gefächert, erstreckt es sich doch über die ganze Bandbreite der Wirtschafts- und Sozialgeschichte. Die Unternehmer- und Unternehmensgeschichte blieb ein Schwerpunkt, seine Einführung „Unternehmensgeschichte“ ist bis heute ein viel gelesenes Handbuch. Empirische Arbeiten zur vergleichenden Bergbaugeschichte, insbesondere zum Bergbau und zur Schwerindustrie Schlesiens im 19. Jahrhundert, gehörten auch dazu. Mit Beiträgen zur historischen Arbeitsmarktforschung betrat er zum Teil Neuland, zog neue Quellen heran (z.B. Zeitungsanzeigen) und erweiterte das Thema Arbeitsmarkt um machtpolitische Überlegungen. Arbeitsmarktstrukturen und Arbeitsbedingungen stellten dann auch einen zweiten Schwerpunkt dar. Mit Beiträgen zur vergleichenden Industrialisierungsgeschichte (,Umstrittene Revolutionen“), zur deutschen Industrialisierungsgeschichte („Gewerbe und Industrie im 19. und 20. Jahrhundert“) und noch mehr mit seiner „Entstehung der modernen Volkswirtschaft“ bot er gut lesbare Einführungen. Zudem nahm er mit dogmengeschichtlichem Interesse die Entwicklung der Wirtschaftswissenschaft als Disziplin in den Blick. Hierzu zählten seine „Geschichte des ökonomischen Denkens“ (2012) und eine sympathisierende Studie über den Ökonom Oskar Stillich (1872-1945). 
Seine Arbeiten zeichneten sich durch gründliche Literatur- und Quellenkenntnisse aus. Selten stand in seinen Texten eine empirische Aussage ohne Beleg. Obwohl er der älteren Wirtschaftshistoriografie kritisch gegenüber stand, wusste er ihre empirische Leistung auch positiv zu würdigen: so seine Einschätzungen zur These „Niedergang des Handwerks“ oder zur Rolle des Preußischen Staates im Industrialisierungsprozess.

Ihm war auch die Bedeutung sinnvoller Quantifizierung wirtschaftshistorischer Sachverhalte bewusst. Dennoch war er kein Cliometriker, der mit formalisierten Modellen Theorien statistisch prüfte. Gleichwohl verstand er wirtschaftstheoretische Aussagen auf Anhieb und war im Stande, wie in seinen einführenden Lehrbüchern intendiert, theoretisch motivierte Fragestellungen für einen breiteren Leserkreis verständlich darzulegen und die Lesenden für aktuelle Bezugspunkte wirtschaftshistorischer Fragen zu sensibilisieren. Er vertrat auch dezidiert die Ansicht, dass die Ökonomie das primäre theoretische Werkzeug und ordnendes Instrument der Wirtschaftsgeschichte sein sollte. Sein Verständnis der Ökonomie wird deutlich in seiner letzten bedeutenden Publikation: die mit Rainer Fremdling verfasste Geschichte des Rheinisch-Westfälischen Instituts für Wirtschaftsforschung (2018), in der er die Wandlung der Wirtschaftswissenschaft und der Wirtschaftspolitik in Westdeutschland unter dem Druck der nach 1945 obwaltenden Sachzwänge beschreibt und interpretiert.

Nirgends war diese methodologische Orientierung Pierenkempers klarer als bei seinem zähen, aber schließlich erfolgreichen Kampf für die Umwandlung des Jahrbuchs für Wirtschaftsgeschichte: vom Organ der Historiker der Akademie der Wissenschaften der DDR zum einem Publikationsforum moderner Wirtschaftsund Sozialgeschichte auf der Basis westlicher Werte und Forschungsmethoden. Seine abwehrende Argumentation galt nicht so sehr der marxistischen Lehre als einer aus seiner Sicht drohenden kulturgeschichtlichen Verwässerung der ökonomischen Ausrichtung des Jahrbuchs. Er lehnte allerdings die in der DDR praktizierte Verkürzung der Wirtschaftstheorie auf „Politische Ökonomie“ ab. Insgesamt gebührt ihm das Verdienst, durch seine Entschlossenheit und Arbeitsbereitschaft das Jahrbuch mit zunächst bescheidenen Mitteln über die schwierige Anfangszeit zu einer zentralen Stimme der deutschen Wirtschaftsund Unternehmensgeschichte erhoben zu haben.

Schon allein aus seinen Bemühungen um das Jahrbuch wird klar, dass Toni Pierenkemper nicht nur ein Vielschreiber gewesen ist, sondern auch ein Wissenschaftler, dem die institutionelle Basis des Fachs Wirtschafts- und Sozialgeschichte viel bedeutet hat. So war er von 1998 bis 2002 Vorsitzender des Wirtschaftshistorischen Ausschusses des Vereins für Socialpolitik. Dabei initiierte er eine ganze Reihe von neuen Forschungsschwerpunkten, zum Beispiel 
über Arbeit und Arbeitsmarktstrukturen oder über das Konsumverhalten privater Haushalte. Forschungsfelder der älteren Wirtschaftsgeschichte, wie etwa die oberschlesische Schwerindustrie und die vergleichende Bergbaugeschichte, revitalisierte er mit neuen Fragestellungen. Mit diesen Projekten bot er auch seinen Schülern und Schülerinnen die Möglichkeit zur Teilhabe an der Forschung. Dies entsprach dem Prinzip der Einheit von Forschung und Lehre, das ihm als akademischer Lehrer sehr am Herzen lag.

Im Laufe seiner Karriere nahm Toni Pierenkemper natürlich auch häufig an wissenschaftlichen Tagungen teil. An den dabei entstehenden Diskussionen beteiligte er sich gerne, oft leidenschaftlich, auch und besonders wenn sich diese um strittige methodische Fragen drehten. Manche Kollegen werden sich vermutlich gut an die Schärfe erinnern, mit der seine Wortmeldungen gelegentlich bekleidet wurden. Sie reflektierte das hohe Gewicht, das er solchen Diskussionen beimaß, und die Ernsthaftigkeit, mit der er sie betrieb: für ihn ein Mittel der Meinungsbildung, das fast die Bedeutung des geschriebenen Wortes hatte.

Nach meinem Eindruck war er ein effektiver Lehrer, dessen Begeisterung für die eigene Arbeit auch bei seinen Studierenden spürbar wurde und zur Mitarbeit motivierte. Am besten dürften dies seine ehemaligen Schüler und Schülerinnen beurteilen. Sie dürften auch seine freundliche, gesellige Art geschätzt haben, denn, wie mit Kollegen, suchte er gerne mit seinen Studierenden das lockere Gespräch, gelegentlich sogar bei ihm zuhause, nicht selten bei Wochenendausflügen, die oft mit Wanderungen verbunden waren und viel Gelegenheit zu produktiven Zwiegesprächen boten.

Toni Pierenkemper war mein Student - nach meiner Erinnerung erst seit 1968. Von Anfang an hat mir sein bescheidenes, aber doch selbstbewusstes Auftreten gefallen. Wissbegierig arbeitete er sich zielstrebig und schnell in das Fach ein und brauchte wenig Führung. Sein Interesse an einem Studienjahr an der London School of Economics für 1969/70 fand auch deshalb meine volle Unterstützung. Dieses Muster eines Lehrer-Student-Verhältnisses blieb für einige Zeit charakteristisch. In den 1980er Jahren trat an dessen Stelle dann eine kollegiale und freundschaftliche Beziehung, die bis zu seinem Tod hielt. Als das tragische Ende unabwendbar schien, ahnte ich das Ausmaß des Verlustes: eines loyalen Freundes und Kollegen, von dem ich viel gelernt habe - wahrscheinlich mehr als umgekehrt er von mir. Es war mein Glück, ihn gut gekannt und ihm nahegestanden zu haben. Die positive Ausstrahlung seiner Persönlichkeit wird uns allen fehlen. 